\title{
Ghost Imaging at an XUV Free-Electron Laser
}

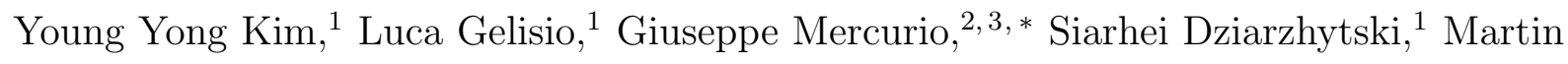
Beye, ${ }^{1}$ Lars Bocklage, ${ }^{1,4}$ Anton Classen,${ }^{5,6}$ Christian David, ${ }^{7}$ Oleg Yu. Gorobtsov, ${ }^{1,}$ Ruslan Khubbutdinov, ${ }^{1,8}$ Sergey Lazarev, ${ }^{1,9}$ Nastasia Mukharamova, ${ }^{1}$ Yuri N. Obukhov, ${ }^{10}$

Benedikt Rösner, ${ }^{7}$ Kai Schlage, ${ }^{1}$ Ivan A. Zaluzhnyy, ${ }^{1}$ 用 Günter Brenner, ${ }^{1}$ Ralf Röhlsberger, ${ }^{1,4}$ Joachim von Zanthier, ${ }^{5,6}$ Wilfried Wurth, ${ }^{1,2,3}$ and Ivan A. Vartanyants ${ }^{1,8, \text { (5 }}$

${ }^{1}$ Deutsches Elektronen-Synchrotron (DESY),

Notkestraße 85, D-22607 Hamburg, Germany

${ }^{2}$ Department Physik, Universität Hamburg, Luruper Chaussee 149, D-22761 Hamburg, Germany

${ }^{3}$ Center for Free-Electron Laser Science, Luruper Chaussee 149, D-22761 Hamburg, Germany

${ }^{4}$ The Hamburg Centre for Ultrafast Imaging, Luruper Chaussee 149, D-22761 Hamburg, Germany

${ }^{5}$ Institut für Optik, Information und Photonik,

Universität Erlangen-Nürnberg, Staudtstraße 1, D-91058 Erlangen, Germany

${ }^{6}$ Erlangen Graduate School in Advanced Optical Technologies (SAOT), Universität Erlangen-Nürnberg, Paul-Gordan-Staudtstraße 6, D-91052 Erlangen, Germany

${ }^{7}$ Paul Scherrer Institut, CH-5232 Villigen PSI, Switzerland

${ }^{8}$ National Research Nuclear University MEPhI (Moscow Engineering Physics Institute), Kashirskoe shosse 31, 115409 Moscow, Russia

${ }^{9}$ National Research Tomsk Polytechnic University (TPU), pr. Lenina 30, 634050 Tomsk, Russia

${ }^{10}$ Theoretical Physics Laboratory, Nuclear Safety Institute, Russian Academy of Sciences, B. Tulskaya 52, 115191 Moscow, Russia

(Dated: December 11, 2019) 


\begin{abstract}
Here we present the results of a classical ghost imaging experiment accomplished at an XUV free-electron laser (FEL). To perform such experiment at an FEL source each x-ray pulse was transmitted through a moving diffuser which created non-correlated speckled beam. This beam was than split in two identical branches by introducing a beam splitter in the form of a transient grating. In one of these branches the sample was positioned. We demonstrate the possibility of image formation, a double bar in our case, in the beam that has never interacted with the sample. By this experiment we extend the quantum optics methodology to the FEL community.
\end{abstract}

\footnotetext{
* Present address: European XFEL GmbH, Holzkoppel 4, D-22869 Schenefeld, Germany

$\dagger$ Present address: Department of Materials Science and Engineering, Cornell University, Ithaca, NY 14850, USA

$\ddagger$ Present address: University of California, San Diego, La Jolla, CA 92093, USA

$\S$ Corresponding author: ivan.vartaniants@desy.de
} 


\section{INTRODUCTION}

With the advent of the laser in the 1960s the new domain of quantum optics emerged [1], which became one of the most dynamic fields of optical science using visible light [2]. Within quantum optics the subject of quantum imaging, based on utilizing correlation techniques, is presently evolving particularly rapidly [3 22] [see for review [23]]. Employing techniques of quantum imaging may lead to an enhanced resolution that can, in principle, overcome Abbe's fundamental limit of classical optics [12, 15, 16, 19].

Recently developed x-ray free-electron lasers (XFELs) [24] are unique sources of bright, femtosecond x-ray radiation. Operation of most of them is based on the self-amplified spontaneous emission (SASE) process [25]. While they possess high 1-st order coherence [26 28], analysis of the 2-nd order coherence shows that SASE FELs statistically behave similar to chaotic sources [29 31]. One important exception from this behavior is the externally seeded FEL FERMI which produces laser-like radiation and exhibits 1-st as well 2-nd order coherence [32].

One exceptional property of the XFEL sources is their high degree of degeneracy, which is the number of photons in a single spatial and temporal mode. Our analysis showed that it is as high as $10^{9}$ at SASE FELs [26, 27, 29] and reaches value of $10^{11}$ for externally seeded FELs [32. That makes them different from synchrotron sources where this parameter is of the order of $10^{-2}$ [33]. Such high values of degeneracy parameter open the way for quantum optics experiments at XFEL sources, as it was previously explored with conventional lasers.

One of the schemes proposed and developed in the domain of quantum imaging is the socalled ghost imaging (GI) technique [4, 7] [see for review [13]]. The idea is based on parallel measurement of two correlated beams [see Fig. 1(a)], where one beam passes through the object and the transmitted light is detected using a bucket detector, whereas the other beam is freely propagating and recorded using a pixelized detector. The reconstructed object results from the coincidence measurements performed between each pixel of the pixelized and bucket detectors. Initially it was assumed that GI needs quantum correlations between the two beams [4], but later it was realized that measurements can also be performed using classically correlated coherent light beams [7], pseudothermal light [8 10], or even thermal sources [11]. Recently, it has been demonstrated that GI can also be implemented using hard $\mathrm{x}$-ray radiation from synchrotron sources [17, 18, 20] and even tabletop x-ray source [21]. At 
the same time, performing GI experiment at an FEL source has many striking advantages in comparison to GI experiments at the limited photon flux sources [see detailed discussion in Appendix A].

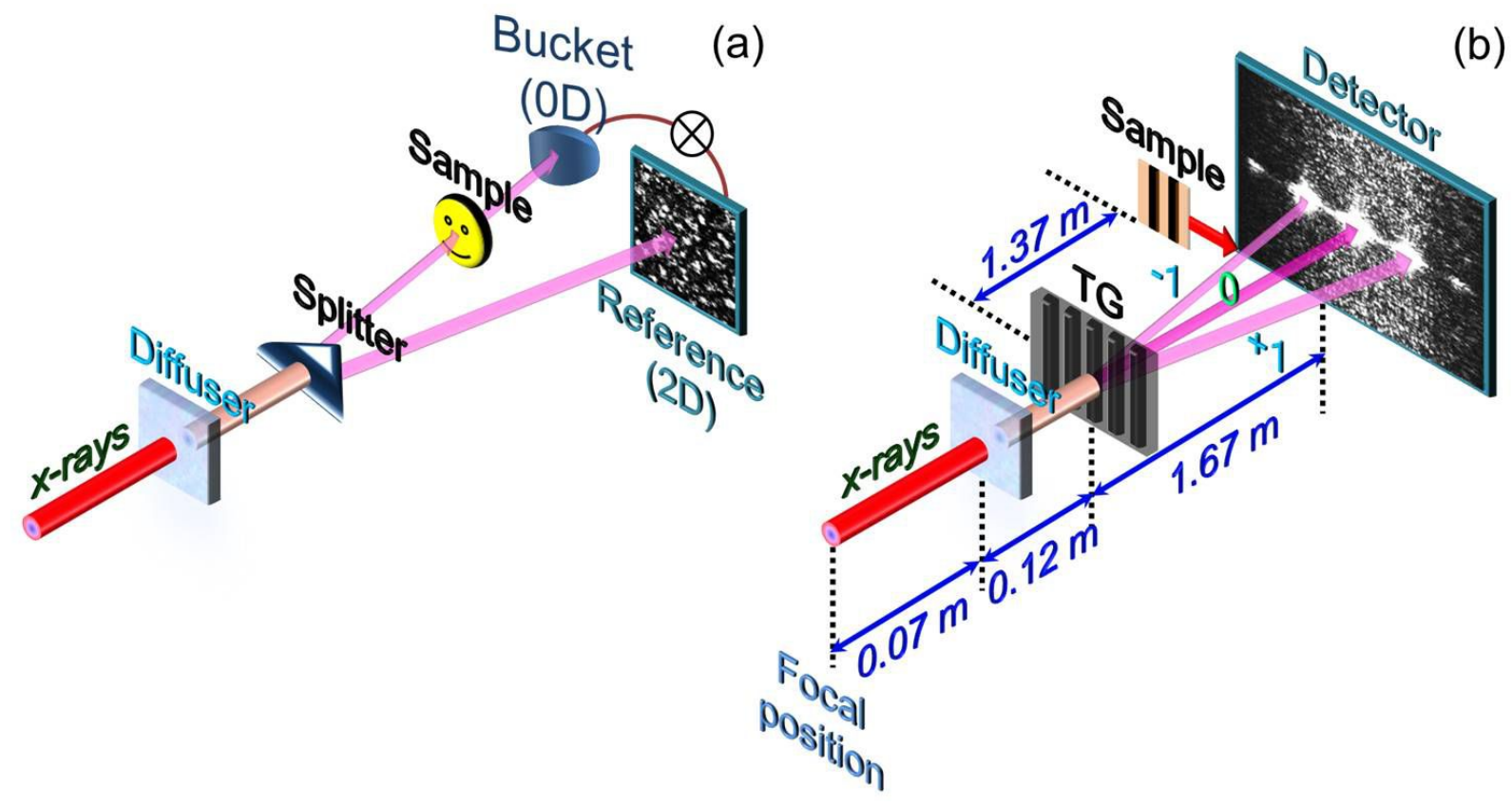

FIG. 1. Conceptual layout of a ghost imaging experiment (a) and schematic setup used at FLASH, which allowed successful ghost imaging using an FEL beam (b).

GI with thermal light sources is based on correlating speckles, i.e. intensity fluctuations of the incident beam, which can be produced either by diffraction of coherent light by a diffuser [34, 35], or utilizing the natural fluctuations of thermal light, as in the original experiment by Hanbury Brown and Twiss (HBT) [36, 37]. The correlation of the intensity in the two arms forms the ghost image $G(x, y)$

$$
G(x, y)=\frac{\left\langle\Delta I_{B} \cdot \Delta I_{R}(x, y)\right\rangle_{M}}{\sqrt{\left\langle\Delta I_{B}^{2}\right\rangle_{M}\left\langle\Delta I_{R}^{2}(x, y)\right\rangle_{M}}}
$$

where $\Delta I_{B, R}=I_{B, R}-\left\langle I_{B, R}\right\rangle_{M}, I_{B}$ is the bucket signal (the total intensity transmitted through the sample), $I_{R}(x, y)$ is the intensity collected at each pixel of the reference detector (which is normalized by $\sum_{x, y} I_{R}(x, y), I_{B}$ ). Intensities $I_{R}(x, y)$ are collected for each individual FEL pulse, and $\langle\ldots\rangle_{M}$ expresses the average over $M$ realizations. Here, we report on the realization of a GI experiment performed in the extreme ultraviolet (XUV) range at an FEL source. 


\section{EXPERIMENT}

For our GI experiments we employed the PG2 beamline of the FLASH facility [38], using the single bunch mode with $10 \mathrm{~Hz}$ repetition rate. The beam from the undulator with average pulse energy of $21 \mu \mathrm{J}$ went through a Xe gas absorber at $3 \times 10^{-2}$ mbar reducing the pulse power by about two orders of magnitude and thus avoiding radiation damage of the detector. The transmitted (0th order) beam from the monochromator at a wavelength of $13.4 \mathrm{~nm}$ (92.5 eV photon energy) had a bandwidth of $\sim 1 \%$ and a footprint of approximately $50 \times 50 \mu \mathrm{m}^{2}$ full width at half maximum (FWHM) at the focal position, $1.86 \mathrm{~m}$ in front of the detection position [39]. The end-station, consisting of a diffuser, transient grating (TG), sample, and detector, is schematically shown in Fig. 1(b). As mentioned, GI at classical sources relies on a varying speckle pattern, used to probe the sample. In our experiment, the speckles were generated by coherently illuminating a diffuser made of silica nanospheres of approximately $200 \mathrm{~nm}$ in diameter [19]. The diffuser was continuously moved such that each FEL pulse was impinging on a different transverse position of the diffuser, in this way the speckle pattern effectively varied from pulse to pulse. As a sample we employed a two-slit structure with slit bars of $200 \mu \mathrm{m}$ width and $1.5 \mathrm{~mm}$ height separated by $200 \mu \mathrm{m}$. It was prepared by electroplating $300 \mathrm{~nm}$ thick cobalt [with transmission of about $10^{-8}$ at 13.4 $\mathrm{nm}$ radiation wavelength] onto a $100 \mathrm{~nm} \mathrm{Si}_{3} \mathrm{~N}_{4}$ substrate. The sample was installed $1.37 \mathrm{~m}$ downstream of the TG and was illuminated by the first order of the grating.

The intensities of the bucket and reference signal, at the end of the experimental unit, were measured simultaneously by an in-vacuum Andor Ikon charge-coupled device (CCD) composed of $2048 \times 2048$ pixels with $13.5 \times 13.5 \mu \mathrm{m}^{2}$ size each, positioned $0.30 \mathrm{~m}$ downstream from the sample. The detector was operated at a repetition rate of $5 \mathrm{~Hz}$ and triggered by the PG2 beamline fast shutter. The bucket and reference signals were defined as regions of $80 \times 80$ pixels on the CCD. The intensity over the area corresponding to the bucket detector was then integrated to mimic the signal of a large area bucket detector. The direct beam [0th order from the TG] was blocked by the sample holder in order to avoid detector saturation.

The most important ingredient for a successful implementation of GI is that the speckle patterns of both arms of the beam are identical and non-overlapping. To reach this goal, a TG made of a $350 \mathrm{~nm}$ free-standing gold structure with a pitch of $17 \mu \mathrm{m}$ and a slit width of $10.2 \mu \mathrm{m}$ was placed in the beam to produce identical copies of the incoming light. 


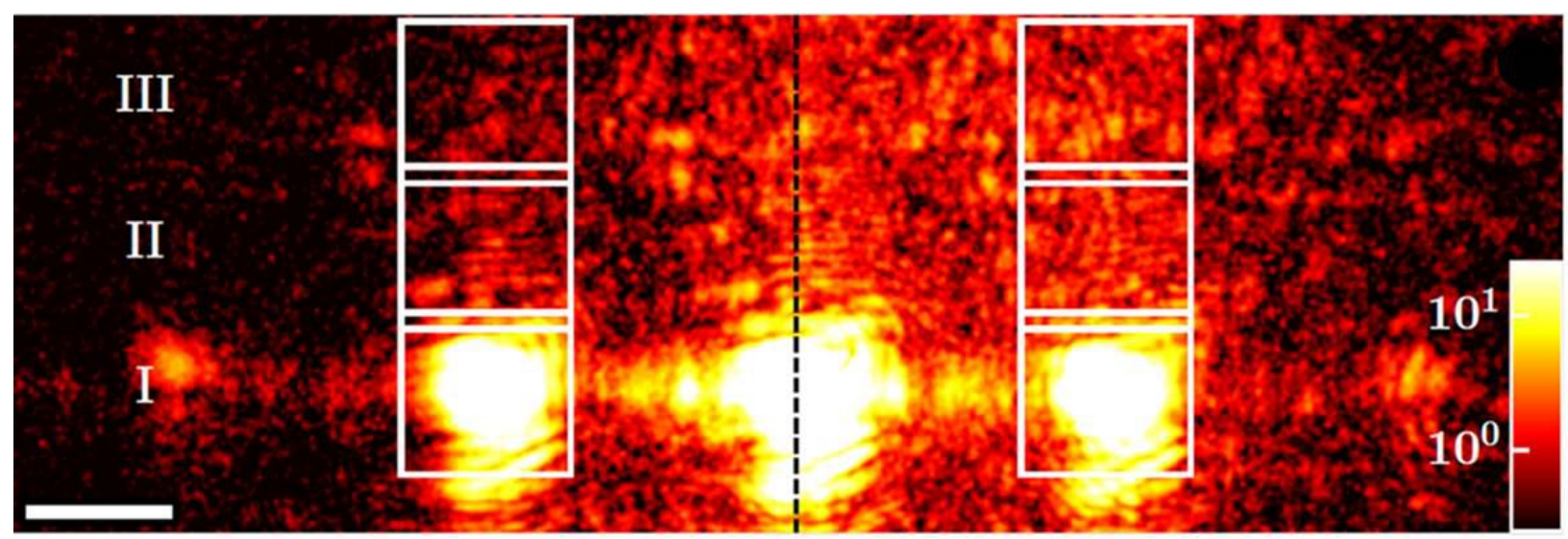

FIG. 2. Single pulse speckle pattern obtained at a selected position of the diffuser. White boxes define different probed positions, the colorbar defines the number of detected photons and the length of the white bar is equal to one millimeter.

\section{RESULTS AND DISCUSSION}

A typical speckle pattern produced by a single FEL pulse and detected in absence of the sample is shown in Fig. 2, with FWHM size of the intensity fluctuations of the order of $100 \mu \mathrm{m}$. Three additional features can be deduced from Fig. 2 and Fig. 4 in Appendix B. First, a stripy structure of the beam extended in the vertical direction, due to specific TG enforcement technology, can be observed. Second, a partial overlap of intensities from different diffraction orders is visible. Third, a small asymmetry in the intensity distribution with respect to the grating symmetry plane of the 0-th order [vertical black dashed line in Fig. 2 was detected. While the latter was possibly caused by a misalignment of the TG, the others resulted from the fact that the TG was not specifically designed for the wavelength of the experiment.

Positions at different vertical distances from the diffraction plane, defined in Fig. 2 as regions I, II, and III, were probed to investigate the effect of the direct beam on the GI reconstruction. Results of correlation analysis at each region are provided in Appendix B. It turned out that we were not able to reconstruct any ghost image at positions I and II due to the presence of a strong background, especially evident when considering the average of intensities over all realizations [see Appendix C]. On the contrary, position III was less affected by the direct beam, and mostly consisted of speckles on top of a background which was the most homogeneous among the three cases [see Fig. 4 in Appendix C]. 
By implementing Eq. (1) to 20,000 realizations of position III, we obtained the ghost image shown in Fig. 3(a). While, this image resembled the double bar shape illustrated in Fig. 3(c), to improve the result, we further reduced the inhomogeneity of the background and increased the speckle contrast by means of an intensity normalization procedure [see Appendix D]. The result of this normalization, applied separately to each realization of the reference region, clearly exhibits a more uniform and flat time-averaged background with respect to the original one [see Fig. 6 in Appendix D]. After applying Eq. (1) and subtracting from each diffraction pattern the background shown in Fig. 6(e) in Appendix D, we obtained the ghost image displayed in Fig. 3(b). The resemblance of the latter with the expected result [see Fig. 3(c)] demonstrates the importance of a proper consideration of the background. To translate the qualitative visual assessment of Figs. 3(a,b) to a quantitative estimation, we projected the intensities along the vertical direction of the detector, obtaining a onedimensional curve. In Fig. 3(d) we compare the projections of the near field image of the object, measured using the bucket detector as a pixelized detector and shown in Fig. 3(c), and its ghost images. While the curve obtained without intensity normalization [Fig. 3(a)] does not perfectly fit the bucket line, the one with normalization [Fig. 3(b)] nicely maps the expected result.

Apart from the inhomogeneity of the background, additional aspects contributed to the reduction of the maximum resolution achievable in our experiment. First, speckle patterns corresponding to different TG orders were not confined enough in space and thus overlapped [see Figs. 2]. To overcome this obstacle and increase the resolution, an improved beam splitter with larger beam separation angle should be used in future experiments. Second, the size of the speckles produced by the diffuser should ideally match the size of the detector pixel, which in turn should be as small as possible for higher resolution.

To assess the role of the speckle size on the resolution in GI, we performed simulations varying this parameter and using the wavelength, sample size, and geometry as in our experiment [see Fig. 1(b)]. In particular, we generated 20,000 realizations of a random speckle pattern using random phase field approach [40], with FWHM size of the speckles ranging from $30 \mu \mathrm{m}$ to $200 \mu \mathrm{m}$ at the detector. We then propagated these fields to the reference and bucket detector positions [see Appendix E]. As illustrated by Fig. 8 in Appendix E, our simulations demonstrate improvement of the spatial resolution when the size of the speckles decreases to values similar to the detector pixel size. 

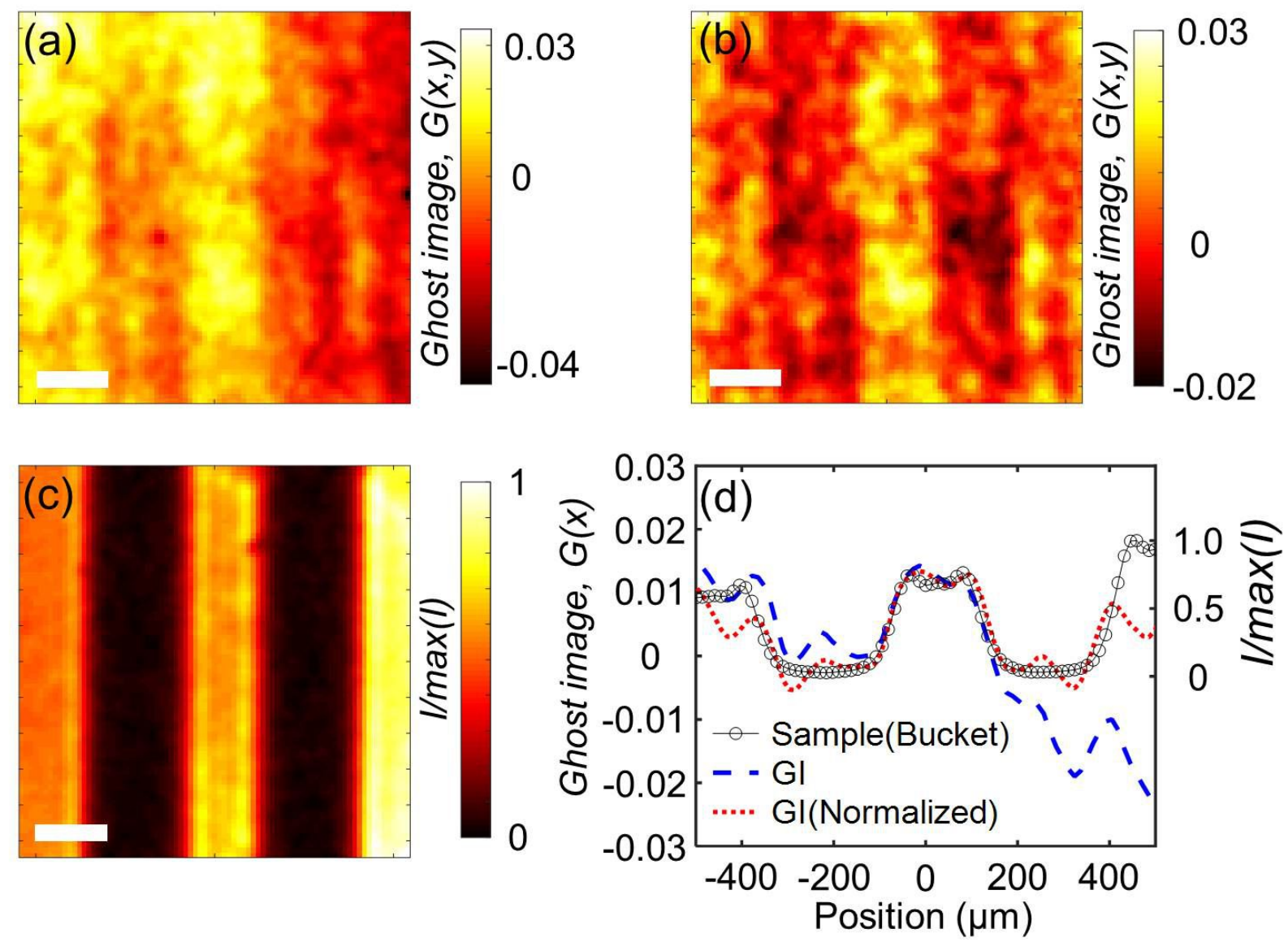

FIG. 3. Results of ghost imaging reconstruction G(x,y) [see Eq. (1D] without (a) and with (b) intensity normalization of reference signal. (c) An averaged image at the bucket detector position. The white scale bar in [(a)-(c)] is $200 \mu \mathrm{m}$. (d) Comparison of intensities projected along the vertical direction in [(a), dashed blue line], [(b), dotted red line] and [(c), black empty dots].

To investigate the effect of photon density on GI reconstructions, we added Poisson noise to the simulated data [see Appendix E]. Since Poisson noise depends only on the incoming number of photons, it is interesting to compare reconstruction results as a function of photons flux, while keeping constant the size of the speckles to $100 \mu \mathrm{m}$. We compare GI results when the number of photons approximate the one available for our experiment [Fig. 9(a)] and cases when it is one [Fig. 9(b)] and two orders of magnitude lower [Fig. 9(c)], like potentially available at other x-ray sources. Clearly, for the greater photon flux we obtained the higher quality of the reconstruction, but even at the lowest photon flux of $50 \mathrm{ph} / \mathrm{pulse}$ the double bar sample is still recognizable. 


\section{SUMMARY}

In summary, we demonstrated ghost imaging at an XUV free-electron laser facility, employing a diffuser to generate a speckle pattern and a transient grating to produce identical copies of the speckle beam. We obtained the ghost image of a double slit structure by computing the correlations from 20,000 frames between a pixelized reference area and the integral of a bucket region. We investigated the effect of an inhomogeneous background on the reconstruction of the ghost image by probing different positions relative to the direct beam. We finally demonstrated a successful reconstruction of the double bar structure for the position characterized by the most uniform background.

With this experiment, we pave the way for ghost imaging at free electron laser facilities. Given the flexibility of the ghost imaging and its potential, this technique, belonging to the realm of quantum based imaging protocols, is expected to become an important tool for imaging in future.

\section{ACKNOWLEDGMENTS}

We acknowledge E. Weckert for fruitful discussions and support of the project and M. Rose for careful reading of the manuscript. We are grateful to the FLASH machine operators and the technical staff at FLASH for excellent FEL conditions. This work was supported by the Helmholtz Associations Initiative and Networking Fund and the Russian Science Foundation grant HRSF-0002. MB is funded through the Helmholtz Association via grant VH-NG-1105.

\section{Appendix A: Comparison between the FEL and synchrotron x-ray measurements}

GI experiments using synchrotron radiation are challenging mostly due to the limited photon flux per synchrotron pulse which strongly degrades the quality of the images [17]. On the other hand, performing such an experiment at an FEL has many striking advantages. First, the pulses produced at FELs have very high intensities [e.g. $10^{9}$ photons per pulse at FLASH FEL [29]], i.e, many orders of magnitude more than what can delivered by synchrotron sources. This is also means that single realization in FEL measurements coincide with the single pulse measurement that potentially reduce the total measurement time. For 
example, our measurements at FLASH took about one hour of accumulation time [20,000 images with $5 \mathrm{~Hz}$ repetition rate]. Second, coincident detection of the two images in the two arms of the splitted beam is inherently provided by the short (femtosecond) pulsed operation of the FEL radiation. And, finally, it is comparably easy to obtain Fourier limited pulses from FELs that provide 100\% contrast in HBT interferometry [29, 30, 32, which leads to strongly enhanced contrast in the GI experiments.

\section{Appendix B: Intensity distribution and correlation of the bucket and reference} beams

An example of the measured intensity distribution in absence of the sample and averaged over 2,115 realizations is reported in Fig. 4(a). Diffraction up to the 5th order is clearly visible, with the beam FWHM size at the first orders being about $340 \mu \mathrm{m}$.

For GI, identical speckle beams must illuminate the sample and the reference area at each pulse. To verify this condition for our experiment we performed cross-correlation analysis of the measured speckle patterns. The general definition of cross-correlation $\gamma(x, y)$ of an arbitrary template $I_{B}(x, y)$ of size $\left(N_{x}, N_{y}\right)$ and a two-dimensional dataset $I(x, y)$ for a single realization can be expressed as

$$
\gamma(x, y)=\sum_{x^{\prime}=1}^{N_{x}} \sum_{y^{\prime}=1}^{N_{y}} I\left(x+x^{\prime}, y+y^{\prime}\right) I_{B}\left(x^{\prime}, y^{\prime}\right)
$$

To account for different illumination conditions, e.g. strong beam gradients, $\gamma(x, y)$ can be zero-normalized [i.e. zero mean value] as [41]

$$
\begin{aligned}
\gamma^{\prime}(x, y)= & \sum_{x^{\prime}=1}^{N_{x}} \sum_{y^{\prime}=1}^{N_{y}} \frac{I\left(x+x^{\prime}, y+y^{\prime}\right)-\mu_{I}(x, y)}{\sigma_{I}(x, y)} \\
& \times \frac{I_{B}\left(x^{\prime}, y^{\prime}\right)-\mu_{B}}{\sigma_{B}},
\end{aligned}
$$

where $\mu_{B}$ is the mean and $\sigma_{B}$ is the standard deviation of the template $I_{B}(x, y)$. The local mean $\mu_{I}$ and the local standard deviation $\sigma_{I}$ of the dataset $I(x, y)$, calculated over the 


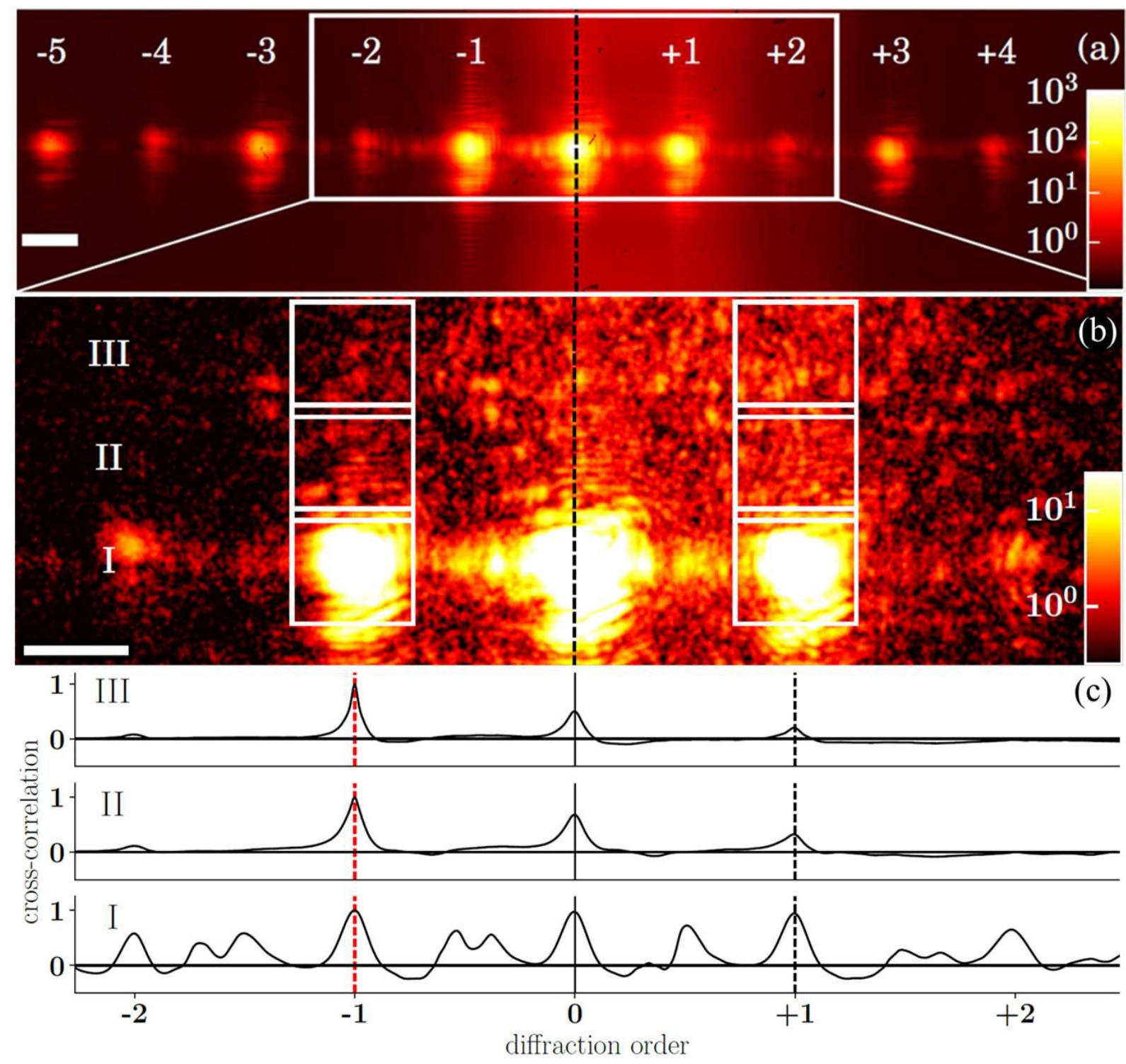

FIG. 4. (a) Time-averaged intensity over 2,115 realizations. (b) Intensity at a selected position of the diffuser, shown to highlight the speckle pattern. White boxes define different probed positions. In $(\mathrm{a}, \mathrm{b})$ the colorbar defines the number of detected photons whereas the length of the white bar equals $500 \mu \mathrm{m}$. (c) Results of the time-averaged zero mean normalized cross-correlation function, ranging from +1 (perfect correlation) to 0 (no correlation). Each of the boxes corresponding to -1-order in (a) is correlated with every detector position and the line through each box center is drawn. The structure of the diffracted beam is especially evident from position I, demonstrating strong correlation for $\pm 1, \pm 2$, and 0 -order as well as half-orders, corresponding to diffraction from the second harmonic in the FEL beam. 
template region $I_{B}(x, y)$, are defined as

$$
\begin{aligned}
& \mu_{I}(x, y)=\frac{1}{N_{x} N_{y}} \sum_{x^{\prime}=1}^{N_{x}} \sum_{y^{\prime}=1}^{N_{y}} I\left(x+x^{\prime}, y+y^{\prime}\right), \\
& \sigma_{I}(x, y)=\frac{1}{N_{x} N_{y}} \sqrt{\sum_{x^{\prime}=1}^{N_{x}} \sum_{y^{\prime}=1}^{N_{y}}\left[I\left(x+x^{\prime}, y+y^{\prime}\right)-\mu_{I}(x, y)\right]^{2}} .
\end{aligned}
$$

The zero-normalized cross-correlation function is defined in the range $[-1,+1]$, where -1 implies anti-correlation, 0 no correlation and +1 full correlation.

To quantify the correlation among different positions on the detector [regions I, II, and III shown in Fig. 4(b)] we implemented the zero mean normalized cross-correlation of the bucket region intensity $I_{B}(x, y)$ at the -1-order, where the sample was positioned, and detected intensity $I(x, y)$. In particular, Fig. 4(c) shows the average zero mean normalized crosscorrelation over 2,115 realizations. To smooth the noise, detected data were first convolved with a Gaussian filter of $1 \times 1$ pixels standard deviation.

The strongest correlation is between the -1-order, illuminating the sample, and the 0order, which was blocked by the sample holder during the experiment to avoid detector saturation. The correlation is also noticeable between the -1 -order and +1 -order, satisfying therefore the necessary condition to perform ghost imaging.

\section{Appendix C: Ghost imaging reconstructions at the positions I and II}

As discussed in the main part of the paper, three regions, defined in Fig. 4(b), were explored during our experiment. While a satisfactory reconstruction was obtained for region III, no image resembling the sample was obtained for regions I and II, as illustrated by Fig. 5 .

\section{Appendix D: Intensity normalization}

To improve our ghost image - in particular to decrease the inhomogeneity of the background and enhancing the contrast of the speckles - we normalized intensities in each realization of the reference area by implementing the additive pattern method developed in [42]. Within this formalism, the image $I(x, y)$ is described as

$$
I(x, y)=\alpha(x, y)+\beta(x, y) I^{\text {corr }}(x, y)
$$



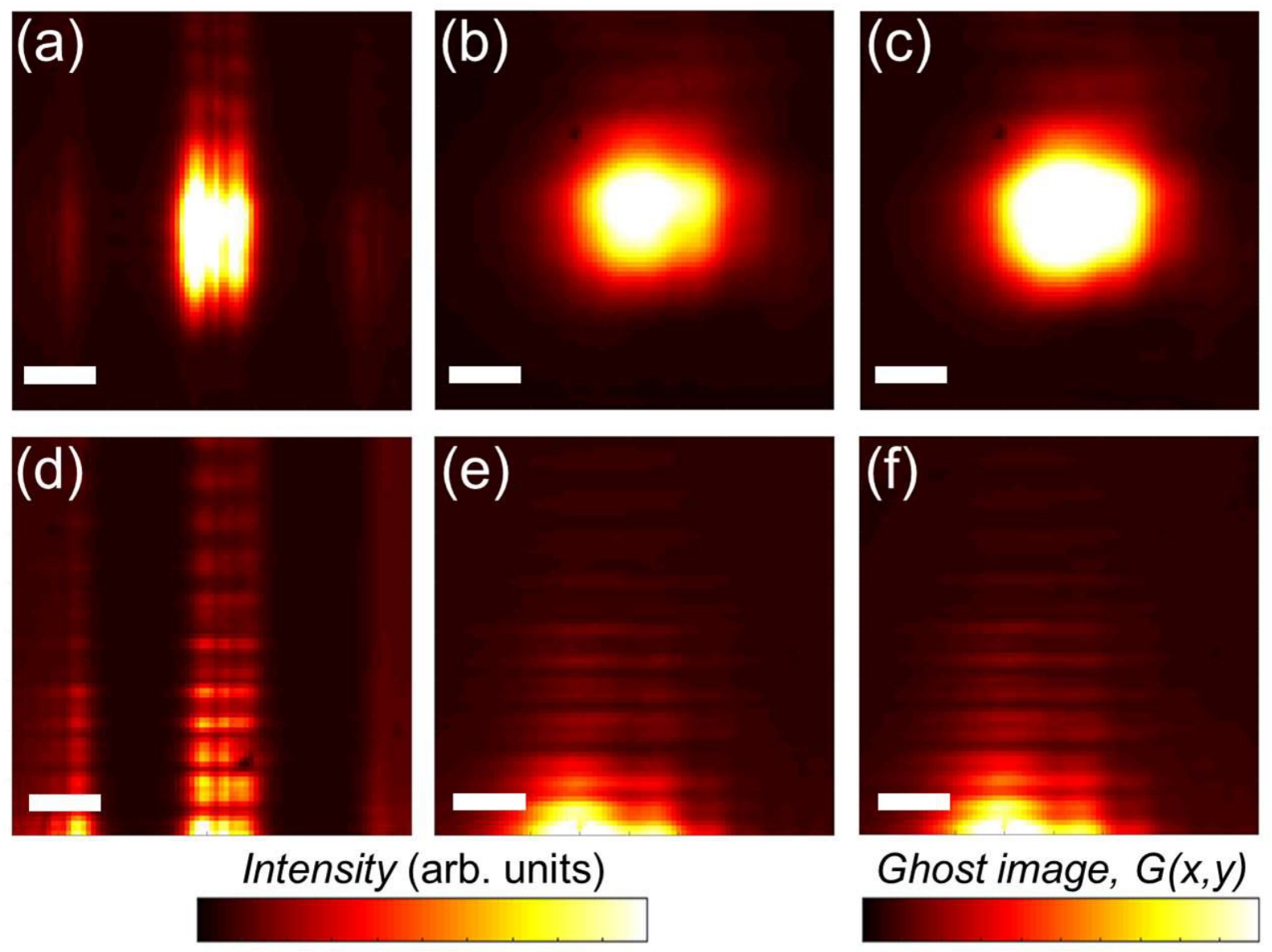

FIG. 5. Results of an averaged intensity and ghost imaging reconstruction at positions I (a,b,c) and II (d,e,f) defined in Fig. 4(b) after 20,000 realizations. An averaged intensity in the bucket (a,d) and reference area (b,e). (c,f) Results of the ghost imaging reconstruction G(x,y) [see Eq. (1)]. The scale bar is $200 \mu \mathrm{m}$.

where $\alpha(x, y)$ and $\beta(x, y)$ are the background and modulation of light respectively, and $I^{\text {corr }}(x, y)$ is the background-corrected intensity used for further analysis.

Elements described above are reported in Fig. 6] [the detailed procedure is discussed in subsection 2.1 of [42]]. In particular, a single realization $I(x, y)$ of the reference detector is shown in Fig. 6(a). From a polynomial fitting of $I(x, y)$, the background $\alpha(x, y)$ [Fig. 6(b)] as well as the light modulation $\beta(x, y)$ [Fig. 6(c)] are extracted. Finally, the result of the procedure, the function $I^{\text {corr }}(x, y)$ which is then used for GI reconstructions instead of $I(x, y)$, is illustrated in Fig. 6(d). 

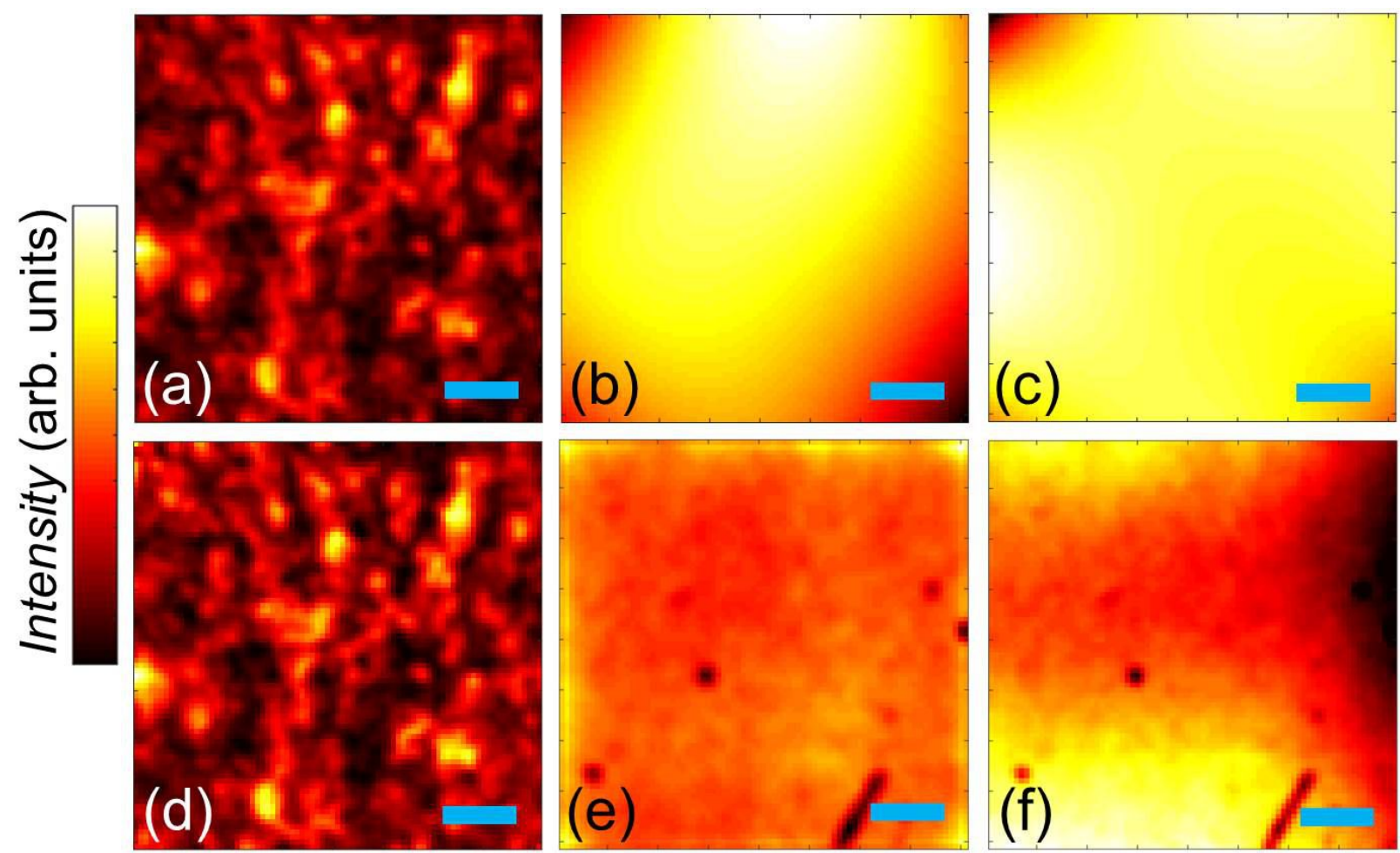

FIG. 6. Intensity normalization procedure in the reference area corresponding to position III in Fig. 4(b). (a) A single realization as measured; (b) the background $\alpha(x, y)$ of (a), extracted by polynomial fitting of (a); (c) the light modulation $\beta(x, y)$; (d) the final result of the procedure, the function $I^{\text {corr }}(x, y)$. The timeaveraged intensity (20,000 realizations) after the normalization procedure (e), compared to the unnormalized averaged-intensity (f). The cyan scale bar is $200 \mu \mathrm{m}$.

\section{Appendix E: Ghost imaging simulations}

Ghost imaging simulations are performed using the same wavelength, sample size, and geometry described in the main part of the paper. However, to avoid background inhomogeneities, the full-width at half-maximum (FWHM) of the beam impinging on the diffuser is considered to be 40 times larger than the experimental one.

\section{Simulations protocol}

To generate a spatially incoherent beam, emulating the one produced by the diffuser, we follow the procedure described in [40]. In particular, we initially generate an electric field having Gaussian amplitude $A_{f}(x, y)$ of $3.5 \mathrm{~mm}$ FWHM and random phase field $\varphi_{f}(x, y) \in$ 
$[-\pi, \pi]$

$$
E_{f}(x, y)=A_{f}(x, y) \exp \left(i \varphi_{f}(x, y)\right)
$$

We then Fourier transform this field to frequency space $\left(f_{X}, f_{Y}\right)$

$$
E_{0}\left(f_{X}, f_{Y}\right)=\mathcal{F}\left[E_{f}(x, y)\right]
$$

and multiply the phase by a Gaussian filter $F_{0}\left(f_{X}, f_{Y}\right)$

$$
E_{f}\left(f_{X}, f_{Y}\right)=F_{0}\left(f_{X}, f_{Y}\right) \frac{E_{0}\left(f_{X}, f_{Y}\right)}{\left|E_{0}\left(f_{X}, f_{Y}\right)\right|} .
$$

The final speckle pattern is obtained as inverse Fourier transform

$$
E_{f}(x, y)=\mathcal{F}^{-1}\left[E_{f}\left(f_{X}, f_{Y}\right)\right]
$$

By this procedure, the variance of the average size of speckles at the diffuser position is the inverse of the variance of the Gaussian filter $F_{0}\left(f_{X}, f_{Y}\right)$ [see an exemplificative realization of the speckle pattern on the diffuser in Fig. 7(a)].

This speckled field $E_{f}(x, y)$ is then propagated using the angular spectrum method [43, 44]. First, the angular spectrum $A_{0}\left(f_{X}, f_{Y}, 0\right)$ is generated from the field $E_{f}(x, y, 0)$,

$$
\begin{aligned}
A_{0}\left(f_{X}, f_{Y}, 0\right)= & \iint E_{f}(x, y, 0) \\
& \times \exp \left(-i 2 \pi\left(f_{X} x+f_{Y} y\right)\right) \mathrm{d} x \mathrm{~d} y .
\end{aligned}
$$

Then, to determine $E_{z}(x, y, z)$ at the distance $z$ from the diffuser $(z=0)$, the following equations are considered

$$
\begin{aligned}
A_{z}\left(f_{X}, f_{Y}, z\right)= & A_{0}\left(f_{X}, f_{Y}, 0\right) \\
& \times \exp \left(i\left(\frac{2 \pi}{\lambda}\right) z \sqrt{1-\left(\lambda f_{X}\right)^{2}-\left(\lambda f_{Y}\right)^{2}}\right) \\
E_{z}(x, y, z)= & \iint A_{z}\left(f_{X}, f_{Y}, z\right) \\
& \times \exp \left(i 2 \pi\left(f_{X} x+f_{Y} y\right)\right) \mathrm{d} f_{X} \mathrm{~d} f_{Y} .
\end{aligned}
$$

According to our experimental geometry, we propagate the reference beam $E_{z}(x, y, z)$ from the diffuser to the detector, at $z=1.79 \mathrm{~m}$ [see Fig. $7(\mathrm{~b})]$.

To account for the presence of the sample, we first propagate the beam from the diffuser to the object $\left[E_{z_{1}}\left(x, y, z_{1}=1.49 \mathrm{~m}\right)\right]$ and then we multiply this amplitude by the sample 

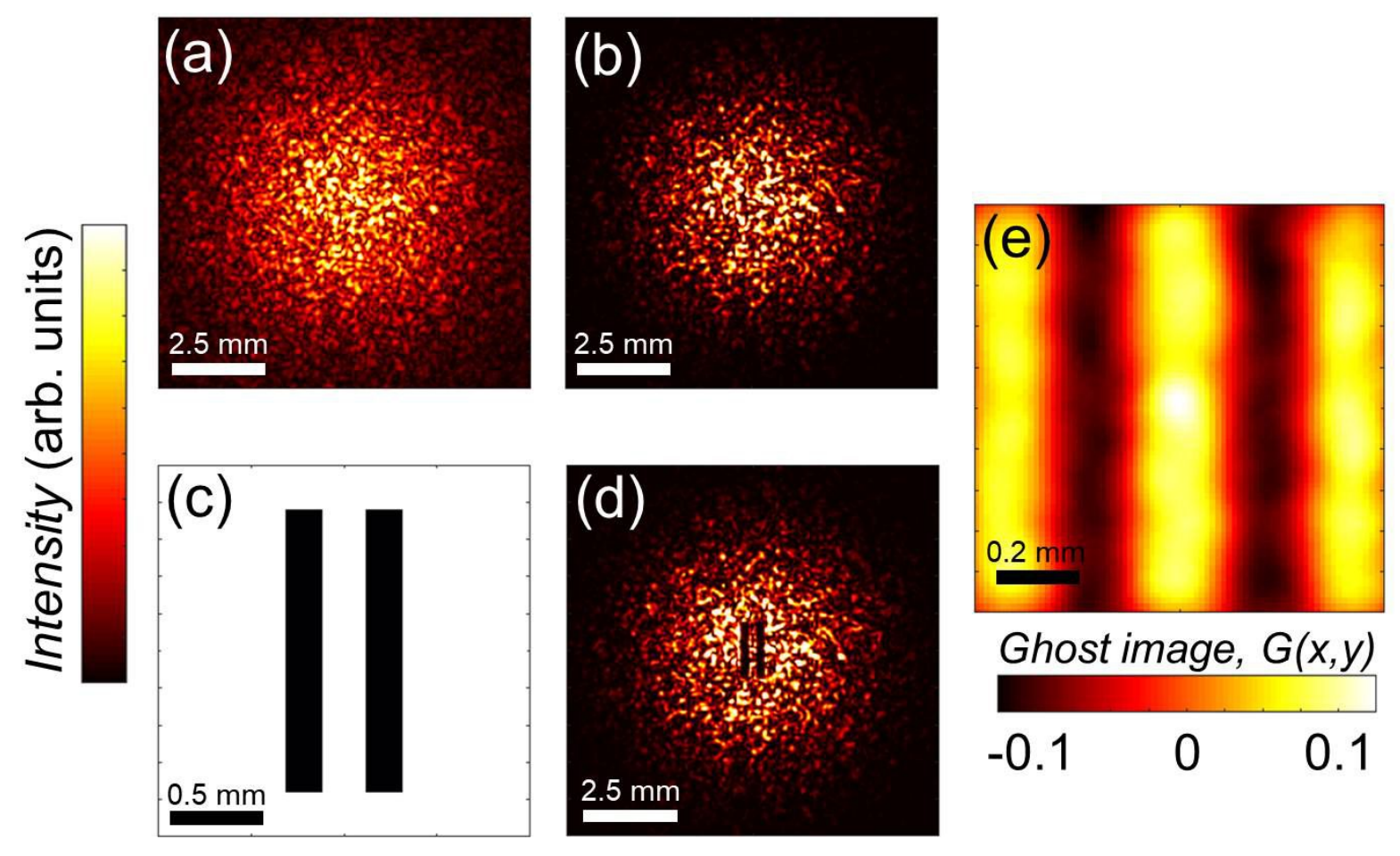

FIG. 7. Simulation procedure. (a) An initial beam consisting of a random speckle patterns; (b) same beam propagated to the reference detector; (c) simulated sample [fully opaque, with the same geometry as the one employed in our experiment]; (d) beam defined in (a) propagated though the sample; (e) result of the ghost imaging reconstruction $\mathrm{G}(\mathrm{x}, \mathrm{y})$ [see Eq. (1]] using a speckle size of $100 \mu \mathrm{m}$ FWHM.

function $O(x, y)$ [illustrated in Fig. $7(\mathrm{c})$ ]. The result is further propagated to the detector $E_{z_{2}}\left(x, y, z_{2}=0.3 \mathrm{~m}\right)$ [Fig. 7(d)]. Finally, ghost imaging calculations are performed on 20,000 realizations generated using the procedure described above. The result of the reconstruction with a speckle size [at the detector position] of $100 \mu \mathrm{m}$ FWHM - corresponding to experimental values - is shown in Fig. 7(e). The whole procedure is implemented in the MATLAB package.

\section{Speckle size, flux and fractionation}

To assess the effect of the size of the speckles on GI spatial resolution, we generated 20,000 realizations of a random speckle pattern, with FWHM size of the speckles ranging from $30 \mu \mathrm{m}$ to $200 \mu \mathrm{m}$. As illustrated by Fig. 8, our simulations demonstrate a strong decrease in resolution when the speckle size approaches the size of the characteristic dimensions of 
the object, which in our case is $200 \mu \mathrm{m}$. On the contrary, the resolution improves when the size of the speckles decreases.
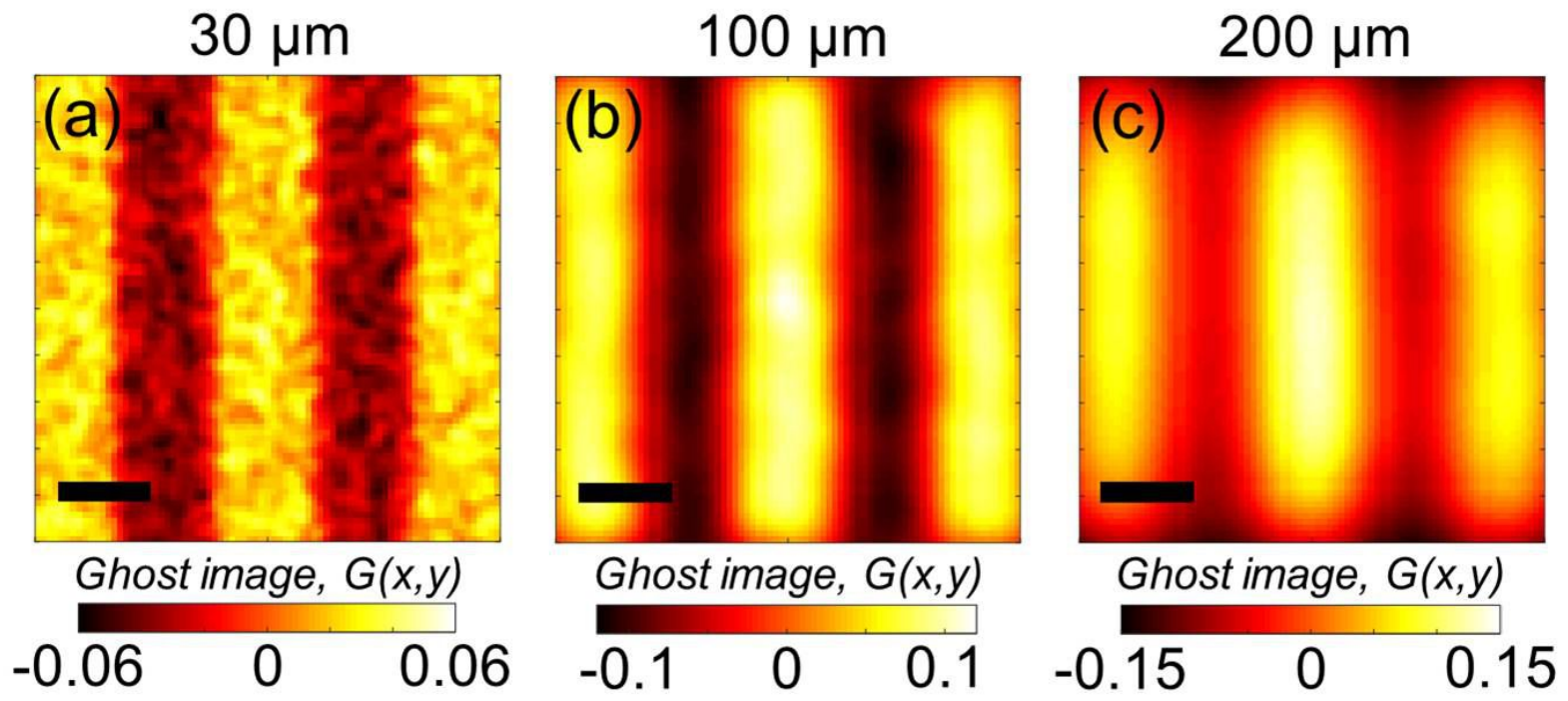

FIG. 8. Ghost imaging simulations G(x,y) [see Eq. [1]] with the FWHM of speckles of (a) $30 \mu \mathrm{m}$, (b) $100 \mu \mathrm{m}$ and (c) $200 \mu \mathrm{m}$.

To address the effect of photon density on GI reconstructions, we started adding Poisson noise to the simulated beam. The noise was determined at detector position from a Poisson distribution considering the total flux detected by the reference and bucket detectors to be of the order of $\Phi=5 \times 10^{3}$ photons per pulse in the conditions of our experiment. This number was estimated for an average pulse integrating the number of counts on the reference region of our detector, consisting of $80 \times 80$ pixels and converting detector counts to photons. Since Poisson noise only depends on incoming number of photons, it is interesting to compare reconstruction results as a function of photons flux, while keeping constant the size of the speckles to $100 \mu \mathrm{m}$. Fig. 9 shows GI results when the number of photons approximate the one available for our experiment and cases when it is one and two orders of magnitude lower, like potentially available at other x-ray sources. Unsurprisingly, the greater the photon flux, the higher the quality of the reconstruction at a given realization.

[1] R. J. Glauber, Phys. Rev. 130, 2529 (1963). 

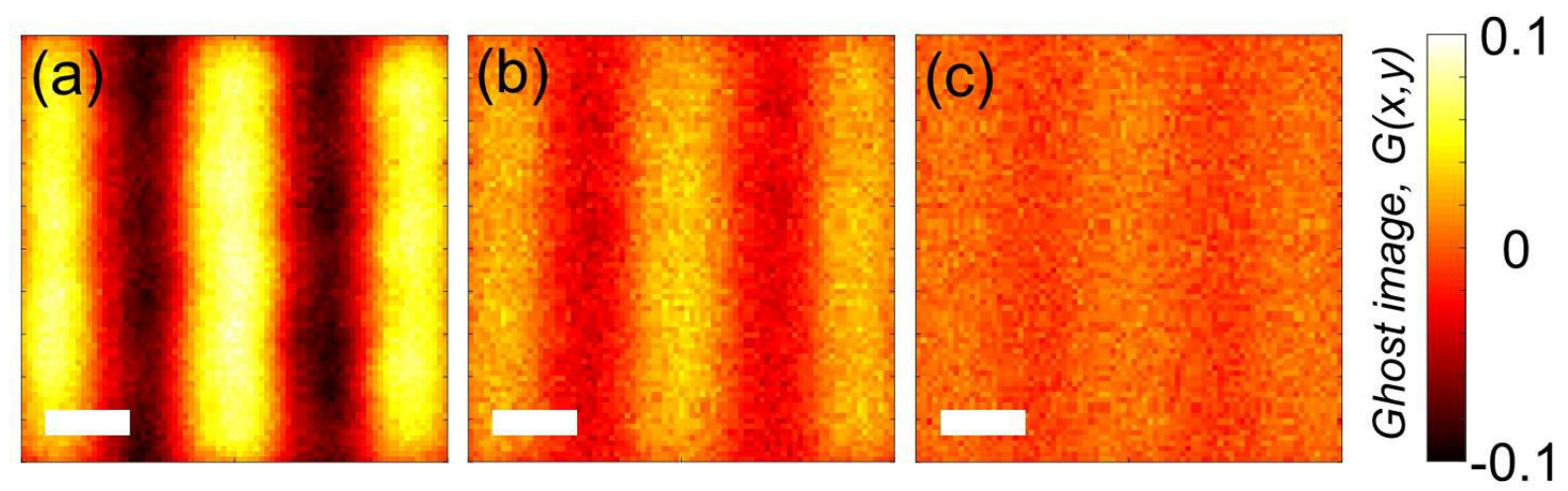

FIG. 9. Ghost image reconstruction G(x,y) [see Eq. [1]] with 20,000 realizations and $100 \mu \mathrm{m}$ speckle size when the number of photons per pulse in both the reference and bucket arm is (a) 5, $000 \mathrm{ph} / \mathrm{pulse}$, (b) 500 $\mathrm{ph} / \mathrm{pulse}$, and (c) $50 \mathrm{ph} /$ pulse. The scale bar corresponds to $200 \mu \mathrm{m}$.

[2] G. Agarwal, Quantum Optics (Cambridge University Press, 2013).

[3] A. V. Belinskii and D. N. Klyshko, J. Exp. Theor. Phys. 78, 259 (1994).

[4] T. B. Pittman, Y. H. Shih, D. V. Strekalov, and A. V. Sergienko, Phys. Rev. A 52, R3429 (1995).

[5] H.-B. Fei, B. M. Jost, S. Popescu, B. E. A. Saleh, and M. C. Teich, Phys. Rev. Lett. 78, 1679 (1997).

[6] A. F. Abouraddy, B. E. A. Saleh, A. V. Sergienko, and M. C. Teich, Phys. Rev. Lett. 87, $123602(2001)$

[7] R. S. Bennink, S. J. Bentley, and R. W. Boyd, Phys. Rev. Lett. 89, 113601 (2002).

[8] A. Gatti, E. Brambilla, M. Bache, and L. A. Lugiato, Phys. Rev. Lett. 93, 093602 (2004).

[9] Y. Cai and S.-Y. Zhu, Phys. Rev. E 71, 056607 (2005).

[10] A. Valencia, G. Scarcelli, M. D'Angelo, and Y. Shih, Phys. Rev. Lett. 94, 063601 (2005).

[11] D. Zhang, Y.-H. Zhai, L.-A. Wu, and X.-H. Chen, Opt. Lett. 30, 2354 (2005).

[12] C. Thiel, T. Bastin, J. Martin, E. Solano, J. von Zanthier, and G. S. Agarwal, Phys. Rev. Lett. 99, 133603 (2007).

[13] B. I. Erkmen and J. H. Shapiro, Adv. Opt. Photon. 2, 405 (2010),

[14] G. Brida, M. Genovese, and I. R. Berchera, Nat. Photon. 4, 227 (2010).

[15] S. Oppel, T. Büttner, P. Kok, and J. von Zanthier, Phys. Rev. Lett. 109, 233603 (2012).

[16] A. Classen, F. Waldmann, S. Giebel, R. Schneider, D. Bhatti, T. Mehringer, and J. von 
Zanthier, Phys. Rev. Lett. 117, 253601 (2016).

[17] D. Pelliccia, A. Rack, M. Scheel, V. Cantelli, and D. M. Paganin, Phys. Rev. Lett. 117, $113902(2016)$.

[18] H. Yu, R. Lu, S. Han, H. Xie, G. Du, T. Xiao, and D. Zhu, Phys. Rev. Lett. 117, 113901 (2016).

[19] R. Schneider, T. Mehringer, G. Mercurio, L. Wenthaus, A. Classen, G. Brenner, O. Gorobtsov, A. Benz, D. Bhatti, L. Bocklage, B. Fischer, S. Lazarev, Y. Obukhov, K. Schlage, P. Skopintsev, J. Wagner, F. Waldmann, S. Willing, I. Zaluzhnyy, W. Wurth, I. A. Vartanyants, R. Röhlsberger, and J. von Zanthier, Nat. Phys. 14, 126 (2018).

[20] D. Pelliccia, M. P. Olbinado, A. Rack, A. M. Kingston, G. R. Myers, and D. M. Paganin, IUCrJ 5, 428 (2018).

[21] A.-X. Zhang, Y.-H. He, L.-A. Wu, L.-M. Chen, and B.-B. Wang, Optica 5, 374 (2018).

[22] S. Li, F. Cropp, K. Kabra, T. J. Lane, G. Wetzstein, P. Musumeci, and D. Ratner, Phys. Rev. Lett. 121, 114801 (2018).

[23] M. Genovese, J. Opt. 18, 073002 (2016).

[24] C. Pellegrini, A. Marinelli, and S. Reiche, Rev. Mod. Phys. 88, 015006 (2016).

[25] E. Saldin, E. V. Schneidmiller, and M. V. Yurkov, The Physics of Free Electron Lasers (Springer-Verlag Berlin Heidelberg, 2000).

[26] I. A. Vartanyants, A. Singer, A. P. Mancuso, O. M. Yefanov, A. Sakdinawat, Y. Liu, E. Bang, G. J. Williams, G. Cadenazzi, B. Abbey, H. Sinn, D. Attwood, K. A. Nugent, E. Weckert, T. Wang, D. Zhu, B. Wu, C. Graves, A. Scherz, J. J. Turner, W. F. Schlotter, M. Messerschmidt, J. Lüning, Y. Acremann, P. Heimann, D. C. Mancini, V. Joshi, J. Krzywinski, R. Soufli, M. Fernandez-Perea, S. Hau-Riege, A. G. Peele, Y. Feng, O. Krupin, S. Moeller, and W. Wurth, Phys. Rev. Lett. 107, 144801 (2011).

[27] A. Singer, F. Sorgenfrei, A. P. Mancuso, N. Gerasimova, O. M. Yefanov, J. Gulden, T. Gorniak, T. Senkbeil, A. Sakdinawat, Y. Liu, D. Attwood, S. Dziarzhytski, D. D. Mai, R. Treusch, E. Weckert, T. Salditt, A. Rosenhahn, W. Wurth, and I. A. Vartanyants, Opt. Express 20, $17480(2012)$.

[28] F. Lehmkühler, C. Gutt, B. Fischer, M. A. Schroer, M. Sikorski, S. Song, W. Roseker, J. Glownia, M. Chollet, S. Nelson, K. Tono, T. Katayama, M. Yabashi, T. Ishikawa, A. Robert, and G. Grübel, Sci. Rep. 4, 5234 (2014). 
[29] A. Singer, U. Lorenz, F. Sorgenfrei, N. Gerasimova, J. Gulden, O. M. Yefanov, R. P. Kurta, A. Shabalin, R. Dronyak, R. Treusch, V. Kocharyan, E. Weckert, W. Wurth, and I. A. Vartanyants, Phys. Rev. Lett. 111, 034802 (2013), ibid Phys. Rev. Lett. 117, 056401 (2016).

[30] O. Y. Gorobtsov, G. Mercurio, G. Brenner, U. Lorenz, N. Gerasimova, R. P. Kurta, F. Hieke, P. Skopintsev, I. Zaluzhnyy, S. Lazarev, D. Dzhigaev, M. Rose, A. Singer, W. Wurth, and I. A. Vartanyants, Phys. Rev. A 95, 023843 (2017).

[31] O. Y. Gorobtsov, N. Mukharamova, S. Lazarev, M. Chollet, D. Zhu, Y. Feng, R. P. Kurta, J.-M. Meijer, G. Williams, M. Sikorski, S. Song, D. Dzhigaev, S. Serkez, A. Singer, A. V. Petukhov, and I. A. Vartanyants, Sci. Rep. 8, 2219 (2018).

[32] O. Y. Gorobtsov, G. Mercurio, F. Capotondi, P. Skopintsev, S. Lazarev, I. A. Zaluzhnyy, M. B. Danailov, M. Dell'Angela, M. Manfredda, E. Pedersoli, L. Giannessi, M. Kiskinova, K. C. Prince, W. Wurth, and I. A. Vartanyants, Nat. Commun. 9, 4498 (2018).

[33] A. Singer, U. Lorenz, A. Marras, A. Klyuev, J. Becker, K. Schlage, P. Skopintsev, O. Gorobtsov, A. Shabalin, H.-C. Wille, H. Franz, H. Graafsma, and I. A. Vartanyants, Phys. Rev. Lett. 113, 064801 (2014).

[34] W. Martienssen and E. Spiller, Am. J. Phys. 32, 919 (1964).

[35] L. E. Estes, L. M. Narducci, and R. A. Tuft, J. Opt. Soc. Am. 61, 1301 (1971).

[36] R. Hanbury Brown and R. Q. Twiss, Nature 177, 27 (1956),

[37] R. Hanbury Brown, Nature 178, 1046 (1956).

[38] K. Tiedtke, A. Azima, N. von Bargen, L. Bittner, S. Bonfigt, S. Dsterer, B. Faatz, U. Frhling, M. Gensch, C. Gerth, N. Guerassimova, U. Hahn, T. Hans, M. Hesse, K. Honkavaar, U. Jastrow, P. Juranic, S. Kapitzki, B. Keitel, T. Kracht, M. Kuhlmann, W. B. Li, M. Martins, T. Nez, E. Plnjes, H. Redlin, E. L. Saldin, E. A. Schneidmiller, J. R. Schneider, S. Schreiber, N. Stojanovic, F. Tavella, S. Toleikis, R. Treusch, H. Weigelt, M. Wellhfer, H. Wabnitz, M. V. Yurkov, and J. Feldhaus, New J. Phys. 11, 023029 (2009).

[39] F. Sorgenfrei, W. F. Schlotter, T. Beeck, M. Nagasono, S. Gieschen, H. Meyer, A. Fhlisch, M. Beye, and W. Wurth, Rev. Sci. Instrum. 81, 043107 (2010).

[40] T. Pfeifer, Y. Jiang, S. Düsterer, R. Moshammer, and J. Ullrich, Opt. Lett. 35, 3441 (2010).

[41] R. Bernstein, "Image geometry and rectification," in Manual of Remote Sensing, Vol. 1, edited by N. C. Robert (American Society of Photogrammetry, 1983) Chap. 21, pp. 875-881.

[42] R. Juarez-Salazar, C. Robledo-Sanchez, F. Guerrero-Sanchez, A. Barcelata-Pinzon, 
J. Gonzalez-Garcia, and A. Santiago-Alvarado, Opt. Lasers Eng. 77, 225 (2016).

[43] J. Goodman, Introduction to Fourier Optics, McGraw-Hill Series in Electrical and Computer Engineering: Communications and Signal Processing (McGraw-Hill, 1996).

[44] K. Matsushima and T. Shimobaba, Opt. Express 17, 19662 (2009). 